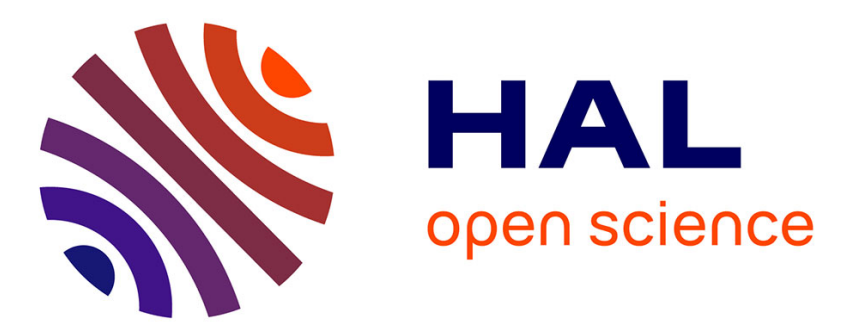

\title{
Mécanismes d'excitation de la conversion rouge-bleu dans les fluorures alcalino-terreux dopés au thulium
}

\author{
M. Bouffard, J. Jouart, G. Mary
}

\section{To cite this version:}

M. Bouffard, J. Jouart, G. Mary. Mécanismes d'excitation de la conversion rouge-bleu dans les fluorures alcalino-terreux dopés au thulium. Journal de Physique IV Proceedings, 1994, 04 (C4), pp.C4-439-C4-442. 10.1051/jp4:19944105 . jpa-00252554

\section{HAL Id: jpa-00252554 https://hal.science/jpa-00252554}

Submitted on 1 Jan 1994

HAL is a multi-disciplinary open access archive for the deposit and dissemination of scientific research documents, whether they are published or not. The documents may come from teaching and research institutions in France or abroad, or from public or private research centers.
L'archive ouverte pluridisciplinaire HAL, est destinée au dépôt et à la diffusion de documents scientifiques de niveau recherche, publiés ou non, émanant des établissements d'enseignement et de recherche français ou étrangers, des laboratoires publics ou privés. 


\title{
Mécanismes d'excitation de la conversion rouge-bleu dans les fluorures alcalino-terreux dopés au thulium
}

\author{
M. BOUFFARD, J.P. JOUART et G. MARY
}

GRSM, Laboratoire d'Energétique et d'Optique, Université de Reims, BP. 347, 51062 Reims cedex, France

Résumé: L'étude spectroscopique et cinétique de l'ion $\mathrm{Tm}^{3+}$ dans les fluorures alcalino-terreux (en particulier $\mathrm{SrF}_{2}$ ) a montré l'existence de relaxations croisées peuplant le niveau réservoir. Toutefois, la comparaison entre les courbes expérimentales et les simulations par ordinateur effectuées à partir d'un modèle à 4 niveaux, suggèrent que les coefficients de relaxation croisée sont insuffisants pour amorcer un processus d'avalanche.

\section{Processus d'excitation de la fluorescence anti-Stokes}

Les processus d'excitation de la fluorescence anti-Stokes sont généralement classés en trois catégories:

absorptions successives,

transfert d'énergie, avalanche[1-4].

Le mécanisme le plus efficace, l'avalanche, est caractérisé par:

l'existence d'un seuil de densité d'excitation au-delà duquel l'intensité de la fluorescence anti-Stokes croît fortement et brusquement de plusieurs ordres de grandeur, réservoir, un temps de montée (réponse à un échelon) supérieur à la durée de vie du niveau

l'existence d'un point d'inflexion très marqué au début de la montée.

Toutefois, les mécanismes réels sont souvent un mélange de deux (voire de trois) de ces processus de base. En particulier, pour l'ion thulium dans les fluorures alcalino-terreux, il est intéressant d'étudier le cas où existent ensemble, une absorption à partir de l'état fondamental et des relaxations croisées peuplant le niveau réservoir, l'excitation étant résonnante entre le réservoir et le niveau fluorescent. Le modèle à trois niveaux permet d'obtenir les expressions analytiques des différentes populations en régime permanent.

\section{Equations de population}

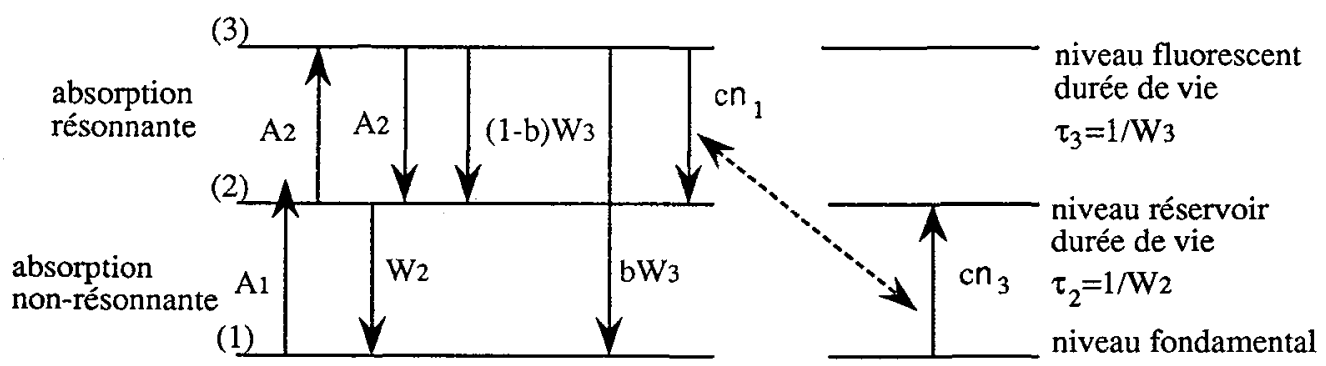

b désigne le rapport de branchement et c le coefficient de relaxation croisée. 


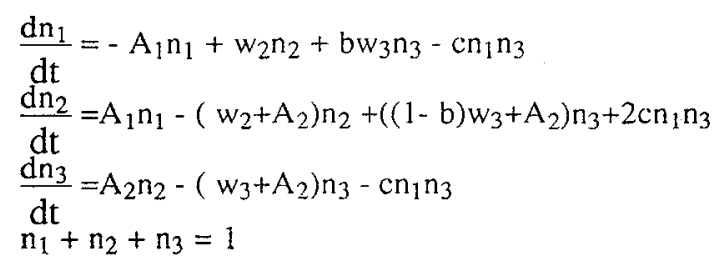

Les calculs des populations, en régime permanent, font apparaitre trois valeurs limites concernant le coefficient $c$ de relaxation croisée et les probabilités d'absorption par unité de temps $\left(A_{2}\right.$ et $\left.A_{1}\right)$.

Pour que le processus d'avalanche soit amorcé, il faut que les trois conditions suivantes soient réalisées simultanément:

$$
\begin{array}{ll}
c>c_{s} \text { avec } & \mathrm{c}_{\mathrm{s}}=\mathrm{bw}_{3}+\mathrm{w}_{2} \\
\mathrm{~A}_{1}<\mathrm{A}_{11} \text { avec } & \mathrm{A}_{11}=\frac{\mathrm{c}-\mathrm{c}_{\mathrm{s}}}{2} \\
\mathrm{~A}_{2}>\mathrm{A}_{2 \mathrm{~s}} \text { avec } & \mathrm{A}_{2 \mathrm{~S}}=\frac{\mathrm{w}_{2}\left(\mathrm{c}+\mathrm{w}_{3}\right)+\mathrm{w}_{3} \mathrm{~A}_{1}}{2\left(\mathrm{~A}_{11}-\mathrm{A}_{1}\right)}
\end{array}
$$

Par conséquent, si le coefficient de relaxation croisée est trop faible ou si l'absorption à partir du fondamental est trop importante, l'avalanche n'aura pas lieu, quelle que soit la valeur de la probabilité d'absorption par unité de temps entre états excités $\left(A_{2}\right)$.

\section{Résultats expérimentaux concernant $\mathrm{SrF}_{2}$}

Nous avons utilisé la technique d'excitation sélective. Le faisceau d'un laser accordable à colorant fonctionnant en continu, est focalisé à l'intérieur du cristal refroidi à l'azote liquide. Dans le domaine visible, la fluorescence antistokes comporte deux groupes de raies:

bleues $\left(480 \mathrm{~nm}\right.$ environ) attribuées à la transition ${ }^{1} \mathrm{G}_{4}-->{ }^{3} \mathrm{H}_{6}$

violettes (450 nm environ) attribuées à la transition ${ }^{1} \mathrm{D}_{2} \rightarrow>{ }^{3} \mathrm{~F}_{4}$,

les niveaux réservoirs étant respectivement ${ }^{3} \mathrm{~F}_{4}$ et ${ }^{3} \mathrm{H}_{4}$. Les sites responsables de la fluorescence antistokes sont de symétrie trigonale et cubique. Dans $\mathrm{CaF}_{2}$, les spectres sont plus difficiles à interpréter car le nombre de sites est plus important.

Plusieurs faits expérimentaux plaident en faveur de l'existence de relaxations croisées à partir des niveaux ${ }^{1} \mathrm{G}_{4}$ et ${ }^{3} \mathrm{H}_{4}$ Dans le domaine spectroscopique, on constate que l'intensité de l'émission violette diminue lorsque la concentration augmente, alors que la fluorescence bleue est renforcée. Dans le spectre d'excitation de la fluorescence proche infrarouge (transition ${ }^{3} \mathrm{H}_{4}->{ }^{3} \mathrm{H}_{6}$ ), il apparait, pour $\mathrm{SrF}_{2}: 1 \% \mathrm{Tm}^{3+}$, une raie d'excitation de la fluorescence bleue alors qu'elle est à peine visible pour $\mathrm{SrF}_{2}: 0,1 \% \mathrm{Tm}^{3+}$. Les spectres d'excitation des deux flurescences blene et violette sont très différents pour $\mathrm{SrF}_{2}: 0,1 \% \mathrm{Tm}^{3+}$. A concentration plus forte ( $1 \%$ et $2 \%$ ), ces spectres sont pratiquement identiques. Dans le spectre d'excitation de la fluorescence violette, les raies correspondant à la transition ${ }^{3} \mathrm{H}_{4} \quad->{ }^{1} \mathrm{D}_{2}$ disparaissent au profit des raies correspondant à la transition ${ }^{3} \mathrm{~F}_{4}->^{1} \mathrm{G}_{4}$ excitant également la fluorescence bleue.

D'autre part, l'étude cinétique montre que les durées de vie des niveaux ${ }^{3} \mathrm{H}_{4}$ et ${ }^{1} \mathrm{G}_{4}$ diminuent quand la concentration augmente. Dans le cristal à $1 \%$, la monté de la fluorescence violette est beaucoup plus rapide pour la raie d'excitation correspondant à la transition ${ }^{3} \mathrm{H}_{4}-\rightarrow{ }^{1} \mathrm{D}_{2}$, que pour la raie correspondant à la transition ${ }^{3} \mathrm{~F}_{4} \rightarrow{ }^{1} \mathrm{G}_{4}$. On constate également que le temps de montée de la fluorescence bleue est supérieur à la durée de vie du réservoir. 


\section{Modèle à quatre niveaux: simulation par ordinateur}

L'écart entre les sous-niveaux montre la nécessité d'un modèle à 4 niveaux:

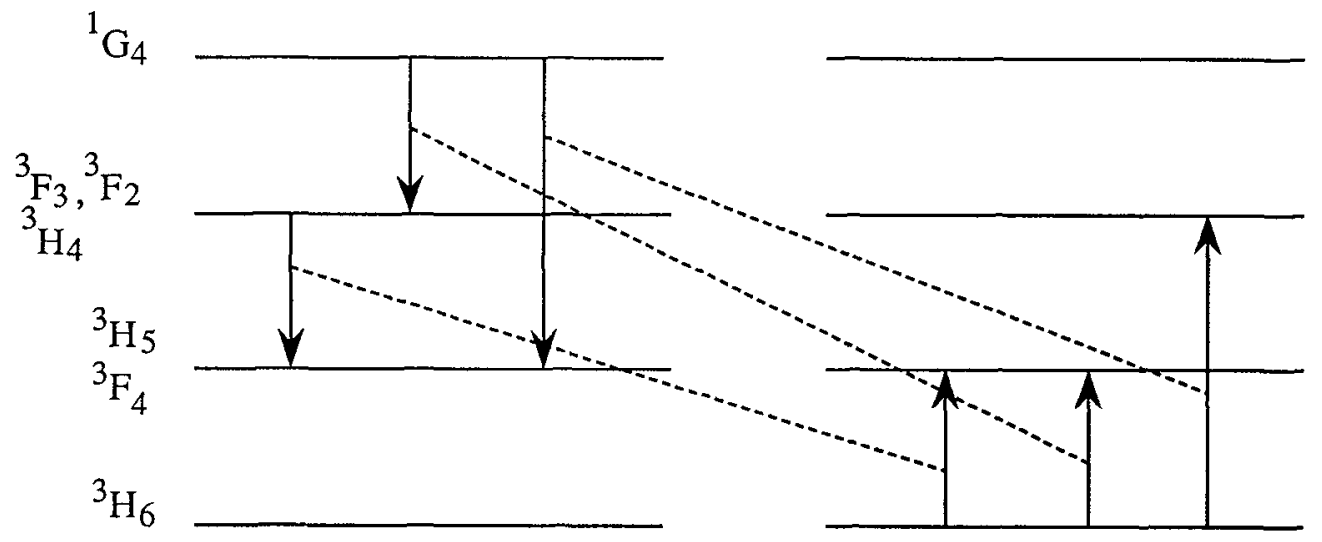

Comme dans le modèle à 3 niveaux, la simulation par ordinateur montre, dans le cas de l'avalanche, une accroissement brusque de la population du niveau fluorescent au passage du seuil (Figure 1).

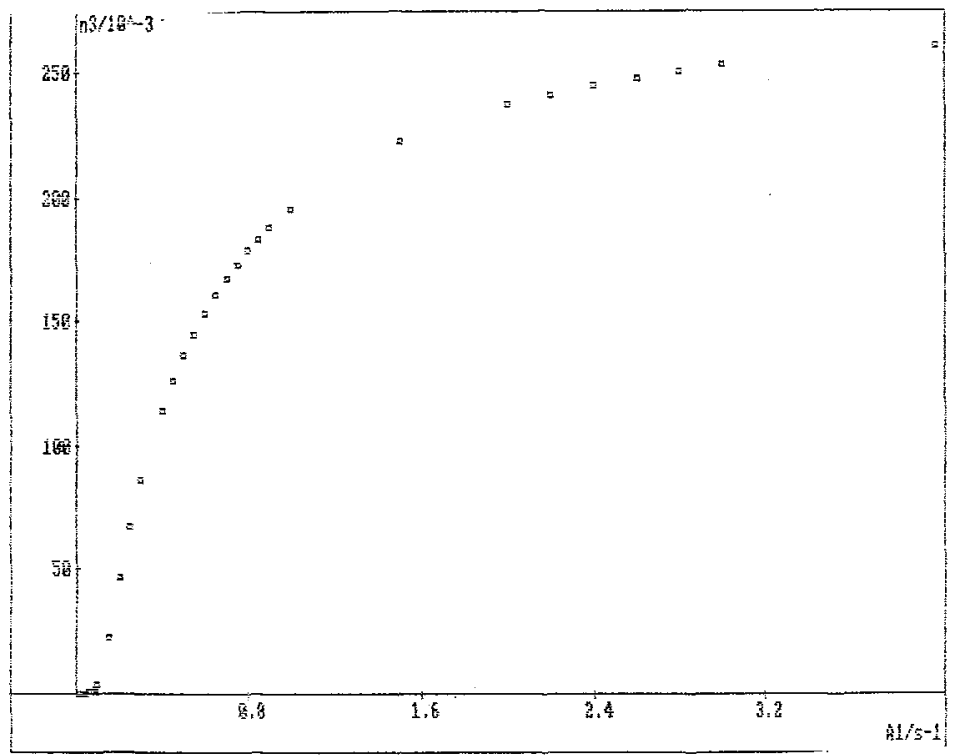

Figure 1 Modèle à 4 niveaux (avalanche), $n_{3}$ : population du niveau fluorescent; $A_{1}$ : probabilité d'absorption par unité de temps

Dans le cas des faibles relaxations croisées, la variation de la population du niveau fluorescent est d'abord quadratique puis linéaire et présente une saturation (Figure 2).

La comparaison avec la courbe expérimentale représentant les variations de l'intensité de la fluorescence en fonction de la puissance excitatrice (Figure 3) suggère que les relaxations croisées restent insuffisantes pour provoquer l'avalanche. 


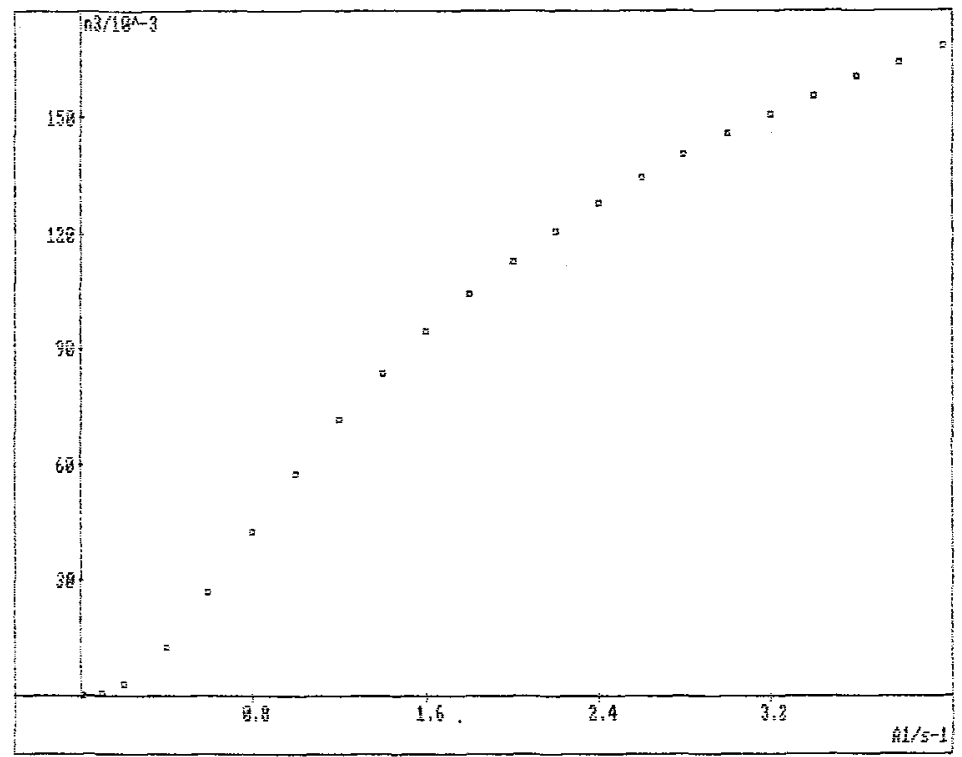

Figure 2 Modèle à 4 niveaux (faibles rclaxations croisćcs), n3: population du niveau fluorescent; $\mathrm{A}_{1}$ : probabilité d'absorption par unitć de temps

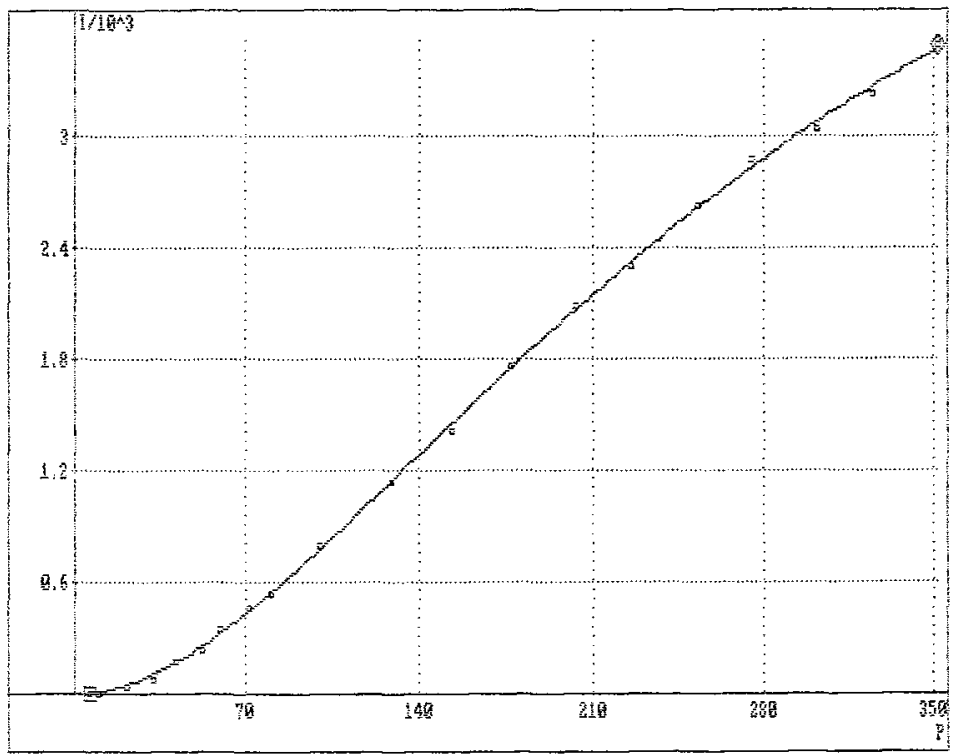

Figure $3 \quad \mathrm{SrF}_{2}: 1 \% \mathrm{Tm}^{3+}$ ćlude en puissance

\section{REFERENCES}

[1] J. S. Chivian, W. E. Case et D. D. Eden, Appl. Phys. Lett. 35(1979)124

[2] W. E. Case, M. E. Koch et A. W. Kueny, J. Lumin. 45(1990)351

[3] T. Hebert, R. Wannemacher, R. M. Macfarlane et W. Lenth Appl. Phys. Lett. 60(1992)2592

[4] M. F. Joubert, S. Guy et B. Jacquier, Phys. Rev. 48(1993) 10031 